\title{
Review
}

\section{Regulation of adenosine levels during cerebral ischemia}

\author{
Stephanie $\mathrm{CHU}^{1}$, Wei XIONG ${ }^{1}$, Dali ZHANG ${ }^{1}$, Hanifi SOYLU ${ }^{1}$, Chao SUN ${ }^{1}$, Benedict C ALBENSI ${ }^{1,2}$, Fiona E PARKINSON ${ }^{1, *}$ \\ ${ }^{1}$ Department of Pharmacology and Therapeutics, University of Manitoba, Winnipeg, Canada R3E OW3; ${ }^{2}$ Division of Neurodegenerative \\ Disorders, St Boniface Hospital Research Centre, Winnipeg, Canada R2H 2 A6
}

\begin{abstract}
Adenosine is a neuromodulator with its level increasing up to 100-fold during ischemic events, and attenuates the excitotoxic neuronal injury. Adenosine is produced both intracellularly and extracellularly, and nucleoside transport proteins transfer adenosine across plasma membranes. Adenosine levels and receptor-mediated effects of adenosine are regulated by intracellular ATP consumption, cellular release of ATP, metabolism of extracellular ATP (and other adenine nucleotides), adenosine influx, adenosine efflux and adenosine metabolism. Recent studies have used genetically modified mice to investigate the relative contributions of intra- and extracellular pathways for adenosine formation. The importance of cortical or hippocampal neurons as a source or a sink of adenosine under basal and hypoxic/ischemic conditions was addressed through the use of transgenic mice expressing human equilibrative nucleoside transporter 1 (hENT1) under the control of a promoter for neuron-specific enolase. From these studies, we conclude that ATP consumption within neurons is the primary source of adenosine in neuronal cultures, but not in hippocampal slices or in vivo mice exposed to ischemic conditions.
\end{abstract}

Keywords: adenosine; cerebral ischemia; ATP; nucleoside transport; hENT1 transgenic mice; hippocampus

Acta Pharmacologica Sinica (2013) 34: 60-66; doi: 10.1038/aps.2012.127; published online 8 Oct 2012

\section{Introduction}

Adenosine is a neuromodulator with receptor-mediated effects. De novo synthesis of adenosine in brain is low ${ }^{[1]}$; thus, adenosine is primarily derived from dephosphorylation of ATP. In physiological conditions, cells salvage adenosine and other nucleosides for nucleotide synthesis. However, in ischemic conditions ATP concentrations drop and adenosine levels rise. Since basal adenosine levels are in the nanomolar range and basal ATP levels are in the low millimolar range, a small percentage drop in ATP can translate into a several fold increase in adenosine levels ${ }^{[2]}$. From these considerations adenosine was termed a "retaliatory metabolite" ${ }^{[3]}$; however, it is evident that adenosine functions in other roles as well.

The purpose of this brief review is to discuss recent findings from mice genetically modified to increase or decrease nucleoside transporter expression. We conclude that the levels and actions of adenosine are influenced by nucleoside transporter expression; however, the experimental preparation and the experimental conditions used modulate the influence of transporter abundance.

\footnotetext{
* To whom correspondence should be addressed.

E-mail fiona.parkinson@med.umanitoba.ca

Received 2012-04-27 Accepted 2012-07-24
}

\section{Adenosine - not merely a retaliatory metabolite}

A common view of adenosine is that it is a retaliatory metabolite and is of particular relevance during hypoxia and ischemia when ATP levels are low ${ }^{[3]}$. Adenosine has effects through activation of members of a family of G-protein coupled receptors, termed $\mathrm{A}_{1}, \mathrm{~A}_{2 \mathrm{~A}}, \mathrm{~A}_{2 \mathrm{~B}}$, and $\mathrm{A}_{3}$. In particular, adenosine $A_{1}$ receptor activity most closely corresponds to that of a retaliatory metabolite, as this receptor produces inhibition of neurotransmitter release secondary to inhibition of calcium channel opening and, in addition, causes post-synaptic inhibition by promoting potassium channel opening ${ }^{[2]}$. The concept of adenosine as a retaliatory metabolite includes the vasodilatation that can result from the activation of adenosine $\mathrm{A}_{2 \mathrm{~A}}$ receptors on vascular smooth muscle, an effect that would serve to enhance delivery of oxygen and glucose to metabolically stressed cells. However, since activation of adenosine $A_{2 A}$ receptors on striatal neurons is associated with enhanced ischemic injury, the view of adenosine as a retaliatory metabolite is insufficient to describe all its actions ${ }^{[4,5]}$. Furthermore, as illustrated by the pharmacological effects of caffeine, a nonselective antagonist of adenosine receptors, it is apparent that adenosine's effects are observed in conditions of physiological levels of oxygen and glucose and not just during conditions of high ATP consumption, such as hypoxia and ischemia. 
As a retaliatory metabolite, adenosine shares the stage with AMP. There is abundant evidence that AMP is an intracellular sensor of energy depletion. As ATP levels fall, AMP levels rise and AMP dependent kinase (AMPK) is activated ${ }^{[6]}$. AMPK is activated by phosphorylation (pAMPK) and it, in turn, phosphorylates a wide range of substrates to activate catabolic pathways and inhibit anabolic pathways ${ }^{[7]}$. AMPK is expressed in neurons and pAMPK is increased in neurons in ischemic brain where it persists during several hours of reperfusion ${ }^{[6]}$. Both neuroprotective and deleterious effects of AMPK inhibition have been reported in stroke studies ${ }^{[6,8]}$. During hypoxia and ischemia, and in tissues with abundant adenosine $\mathrm{A}_{1}$ receptors, it may be that both AMP and adenosine act as retaliatory metabolites, with AMP acting intracellularly via $A M P K$ and adenosine acting extracellularly via its $A_{1}$ receptors.

The view of adenosine as primarily a retaliatory metabolite is also being revised in light of the expanding volume of information about purinergic P2 receptors that utilize ATP and other nucleotides as agonists. The prevalence of these signalling pathways has led to the hypothesis that the effects of adenosine at its receptors are secondary to the effects of nucleotides at P2 receptors ${ }^{[9-11]}$. Depending on the receptor subtype expressed, ATP enhances or inhibits glutamate neurotransmission $^{[12]}$. Thus, it has been demonstrated that ATP can produce inhibitory or excitatory effects, via P2X or P2Y receptors, with subsequent inhibitory effects via $A_{1}$ receptors or excitatory effects via $A_{2 A}$ receptors after metabolism to adenosine $\mathrm{e}^{[10,11]}$.

\section{Nucleoside transporters regulate extracellular adenosine levels}

Nucleoside transporters facilitate the movement of adenosine, and other physiological and chemotherapeutic nucleosides, across biological membranes. Transporter-mediated cellular influx or efflux of adenosine attenuates or enhances, respectively, extracellular levels of adenosine and adenosine receptor activity. Two families of nucleoside transporters have been described, equilibrative and concentrative, with the former expressed by all cell types and the latter localized primarily in absorptive tissues such as epithelial cells ${ }^{[13]}$. Concentrative nucleoside transporters (CNTs) are members of the solute carrier 28 (SLC28) gene family. They are sodium symporters and, thus, mediate cellular influx of nucleosides in the presence of an inwardly directed sodium gradient. Three CNTs (CNT1-3) have been described and CNT2 and CNT3 accept adenosine as a permeant; however, to date a role for CNTs in regulating adenosine levels and adenosine receptor activity has not been demonstrated in CNS tissue ${ }^{[13]}$. Four members of the equilibrative nucleoside transporter (ENT) family, also known as SLC29, have been identified: ENT1-4. ENT1 and ENT2 are bidirectional and, thus, facilitate net cellular influx or efflux of nucleosides, including adenosine, according to the prevailing concentration gradient of permeants. Virtually all cells appear to express ENT1 and/or ENT2. A potent inhibitor, $S$-( $p$-nitrobenzyl)-6-thioinosine (NBTI), is available as a phar- macological tool and as a radioligand for ENT1. No similar highly selective and high affinity probe is available for ENT2; however, non-selective inhibition of ENT1 and ENT2, leading to potentiation of the vasodilatory actions of adenosine, is the pharmacological mechanism of action of the coronary dilators dipyridamole and dilazep. ENT3 and ENT4 are less studied members of the ENT family and their importance in regulating adenosine levels and adenosine receptor activity is poorly characterized. ENT3 has an intracellular localization and is, thus, unlikely to affect adenosine levels and adenosine receptor activity ${ }^{[14]}$. ENT4 is also known as plasma membrane monoamine transporter (PMAT). It is expressed in heart and brain and has relatively low affinity for transport of adenosine and serotonin ${ }^{[15]}$. Interestingly, ENT4 has enhanced activity at acidic $\mathrm{pH}$ relative to physiological $\mathrm{pH}$; thus, it may be of importance in acidotic conditions of ischemia ${ }^{[15]}$.

ENT1 knock out mice were developed to investigate the role of adenosine as a mediator of the pharmacological effects of ethanol ${ }^{[16]}$. These mice exhibited decreased motorincoordination and hypnosis after ethanol administration, which were associated with reduced basal levels of extracellular adenosine and reduced basal activity of adenosine $A_{1}$ receptors in nucleus accumbens ${ }^{[16,17]}$. In contrast to these decreases in adenosine levels in brain, increased blood levels of adenosine were observed in ENT1 knock out mice, relative to wild type mice ${ }^{[18]}$; in further studies, ENT1 knock out mice exhibited ischemic cardioprotection, consistent with enhanced adenosine $\mathrm{A}_{1}$ receptor activity ${ }^{[19]}$.

Considering that extracellular adenosine levels in ENT1 knock out mice appear to be decreased in brain, suggesting intracellular adenosine formation, yet increased peripherally, suggesting extracellular adenosine formation, it appears the pathway for adenosine formation is highly dependent upon the experimental conditions and cells or tissues under investigation. To address the question of whether, or to what extent, adenosine is formed from ATP, the intracellular energy molecule, or from ATP, the extracellular signalling molecule (Figure 1), we have used three different experimental preparations derived from brain: primary cultures of neurons and astrocytes, hippocampal slices and anesthetized mice. Adenosine

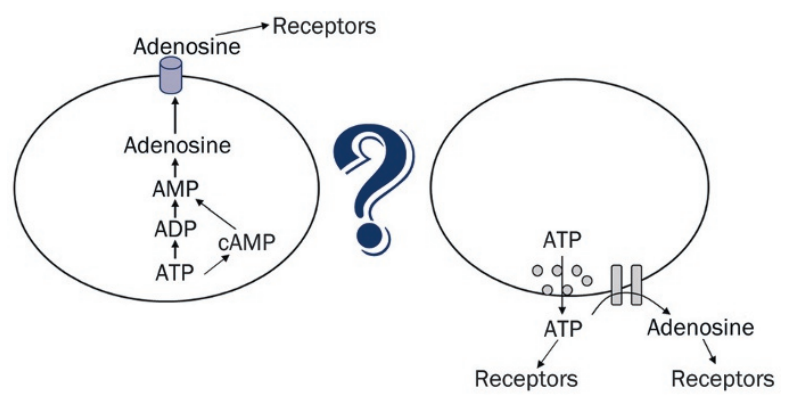

Figure 1. Extracellular adenosine produces receptor-mediated effects. The relative contributions of adenosine formed intracellularly, from ATP the energy molecule, and adenosine formed extracellularly, from ATP the signalling molecule, to the receptor-mediated effects of adenosine are under investigation. 
levels, adenosine $A_{1}$ receptor activity and ischemic infarct sizes were used as outcomes to ascertain whether intracellular or extracellular ATP was the primary source of adenosine during physiological and hypoxic/ischemic conditions.

\section{Cultured neurons, but not astrocytes, release adenosine via ENTs}

To mimic ischemia, rat cortical neurons were treated with $N$-methyl- $D$-aspartate (NMDA), hypoxia, or oxygen-glucose deprivation and increases in extracellular levels of adenosine were measured ${ }^{[20-22]}$. The appearance of adenosine in the extracellular media was inhibited by the equilibrative nucleoside transport (ENT) inhibitor dipyridamole, indicating that adenosine was formed intracellularly during these ATPdepleting conditions, and released from cells via a nucleoside transport system (Figure 2). In contrast, similar experiments were performed with rat cortical astrocytes, but dipyridamole did not affect the appearance of adenosine in the extracellular media $^{[20-22]}$. Instead, $\alpha, \beta$-methylene adenosine diphosphate (AOPCP) decreased the appearance of adenosine ${ }^{[20-22]}$. As AOPCP is an inhibitor of ecto- 5 ' -nucleotidase, these data indicated that astrocytes release adenine nucleotides that are metabolized to adenosine in the extracellular environment.

Co-cultures of rat cortical astrocytes and cortical neurons were tested for the presence of extracellular adenosine following treatment of co-cultures with NMDA, to induce

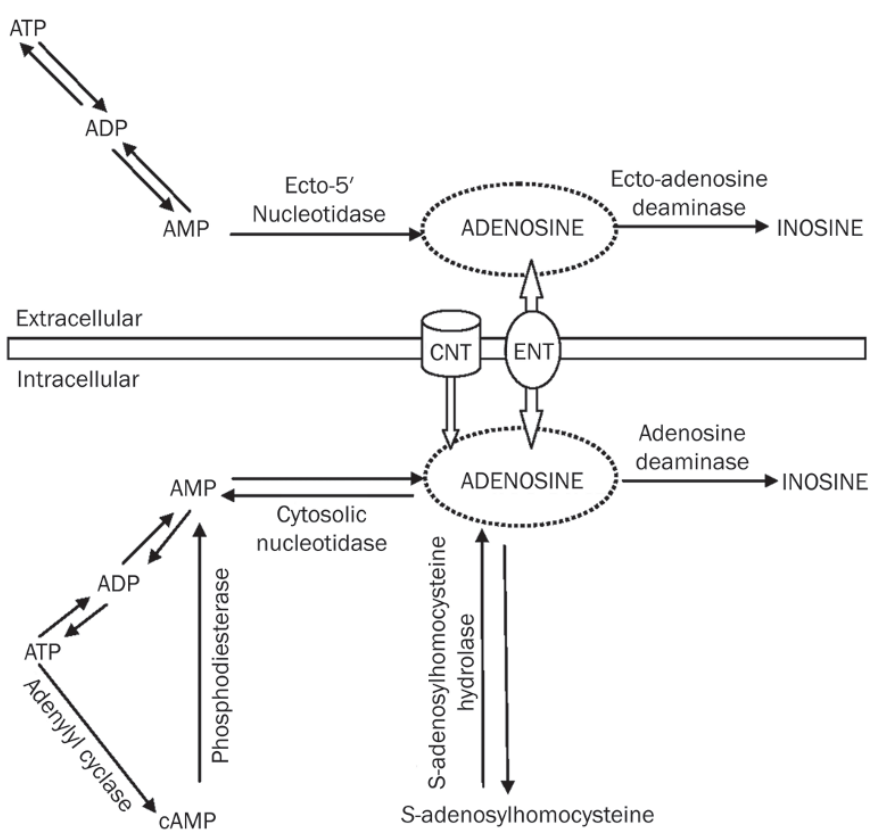

Figure 2. Pathways for adenosine formation. Intracellularly, adenosine is primarily formed by dephosphorylation of ATP, although cAMP and Sadenosyl homocysteine are alternative precursors for adenosine formation. Extracellularly, adenosine is thought to be a product of ATP that is metabolized by a series of ecto-enzymes. Nucleoside transport processes mediate cellular uptake or release of adenosine and other nucleosides, including inosine. Concentrative nucleoside transporters (CNT) and equilibrative nucleoside transporters (ENT) are highlighted. excitotoxicity $^{[21,22]}$. Although NMDA receptors are more abundant on neurons than astrocytes, and NMDA treatment of neurons evokes adenosine release via nucleoside transporters, NMDA treatment of co-cultures induced adenosine release that was inhibited by AOPCP ${ }^{[21,22]}$. These data indicate that the extracellular pathway is predominant when both cell types are present.

\section{Human equilibrative nucleoside transporter 1 (hENT1) transgenic mice}

Given that during excitotoxic conditions, adenosine appears to be released selectively from neurons via a nucleoside transporter mechanism, we developed a transgenic mouse model to further explore adenosine release mechanisms ${ }^{[23]}$. A transgene consisting of the rat promoter region for neuron specific enolase was coupled to the coding sequence of human equilibrative nucleoside transporter 1 (hENT1) and used to develop mice with neuron specific expression of hENT1. We reasoned that ENT facilitate adenosine efflux during events where adenosine receptor mediated effects are a result of intracellular adenosine formation, subsequent to ATP utilization, yet facilitate adenosine influx during events where adenosine receptor mediated effects are a result of extracellular adenosine formation (Figure 2). Since neurons are more sensitive to ischemic injury than astrocytes and show more rapid depletion of intracellular $\mathrm{ATP}^{[20]}$, and since in cell culture rat neurons appear to release adenosine per se during stroke-like conditions ${ }^{[22]}$, we created mice with neuron specific expression of hENT1 $1^{[23]}$.

Using the high affinity and highly selective radioligand $\left[{ }^{3} \mathrm{H}\right] \mathrm{NBTI}$ to radiolabel mouse ENT1 and hENT1, we found high expression of ENT1 proteins in synaptosomes prepared from cortex (Figure 3) and hippocampus ${ }^{[24]}$. Maximum binding site density $\left(B_{\max }\right)$ was increased more than 15 -fold in synaptosomes from transgenic mice (Figure 3) ${ }^{[24]}$. Affinity constants $\left(K_{\mathrm{d}}\right.$ values) were increased 2-fold in samples from transgenic mice.

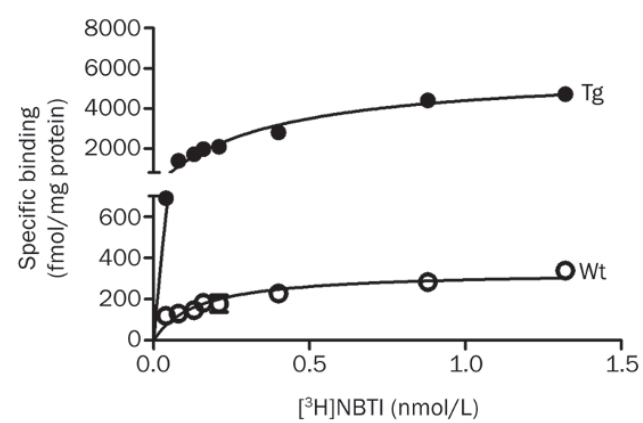

Figure 3. Abundance of human equilibrative nucleoside transporter 1 (hENT1) determined from radioligand binding assays utilizing the selective radioligand $\left[{ }^{3} \mathrm{H}\right] \mathrm{S}$-( $p$-nitrobenzyl)-6-thioinosine $\left(\left[{ }^{3} \mathrm{H}\right] \mathrm{NBTI}\right.$; 0.05-1.2 nmol/L) and synaptosomal preparations from cortex of heterozygous hENT1 transgenic mice (Tg) and wild type littermates (Wt). Assays with cortical synaptosomes were performed using the methods previously published for hippocampal synaptosomes and similar results were obtained ${ }^{[24]}$. 
Cultured neurons, from hENT1 transgenic mice, release adenosine via ENTs

Using cortical neurons cultured from wild type or transgenic hENT1 mice, $\left[{ }^{3} \mathrm{H}\right]$ adenosine uptake into hENT1 neurons was greater than uptake into wild type neurons (Figure 4A). It is of interest that, while there was greater than 15-fold increase in ENT1 density in synaptosomal membranes, this led to only a $40 \%$ increase in $\left[{ }^{3} \mathrm{H}\right]$ adenosine uptake into cultured neurons. However, cellular accumulation of adenosine is determined by transport across the plasma membrane followed by intracellular trapping, with the latter as the rate limiting process. Low activity of adenosine kinase, which has been reported to be an astrocytic enzyme ${ }^{[25]}$, is likely to limit the intracellular accumulation of $\left[{ }^{3} \mathrm{H}\right]$ adenosine by neurons.
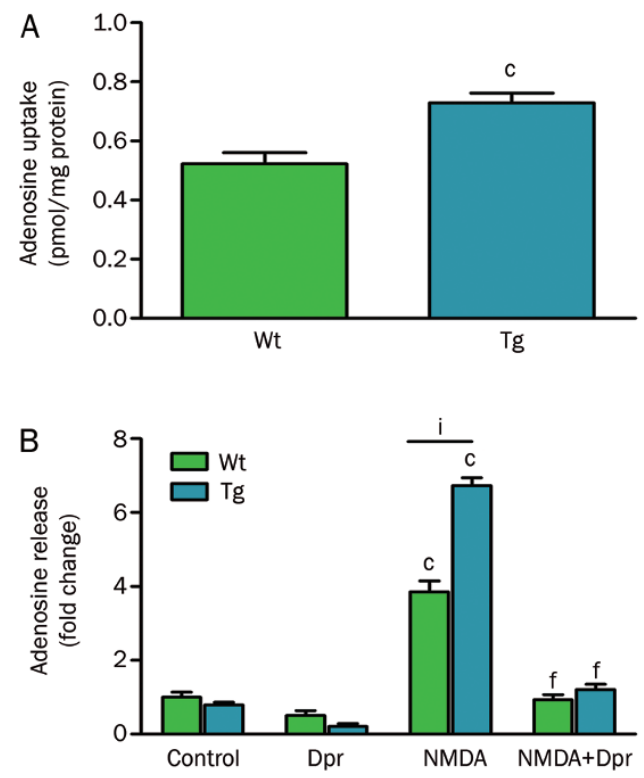

Figure 4. Adenosine uptake $(A)$ and release $(B)$ from cultured cortical neurons from wild type (Wt) or hENT1 transgenic (Tg) mice. (A) $\left[{ }^{3} \mathrm{H}\right]$ Adenosine $(1 \mu \mathrm{mol} / \mathrm{L} ; 0.1 \mu \mathrm{Ci})$ uptake was performed at $22^{\circ} \mathrm{C}$ using primary cultures of neurons (11-15 d in vitro) using an assay volume of $0.5 \mathrm{~mL}$ and an uptake interval of $1 \mathrm{~min}$. Non-specific uptake was determined with dipyridamole (Dpr; $30 \mu \mathrm{mol} / \mathrm{L})$. For further details on methods, see ${ }^{[36]}$. ${ }^{\mathrm{c}} \mathrm{P}<0.01$; $t$-test. (B) Neurons were incubated for $30 \mathrm{~min}$ at $37^{\circ} \mathrm{C}$ with $\left[{ }^{3} \mathrm{H}\right]$ adenine to radiolabel intracellular ATP, then treated with buffer, Dpr (30 $\mu \mathrm{mol} / \mathrm{L}$ ), $N$-methyl-D-aspartate (NMDA; $100 \mu \mathrm{mol} / \mathrm{L}$ ) or the combination of Dpr and NMDA for $30 \mathrm{~min}$ at $37^{\circ} \mathrm{C}$ to evoke release of purines. $\left[{ }^{3} \mathrm{H}\right]$ Purines were separated by thin layer chromatography and quantified by scintillation spectroscopy. For additional details on methods, see ${ }^{[22]}$. Data are mean \pm SEM $(n \geq 8)$ and are expressed as $\mathrm{pmol} / \mathrm{mg}$ protein (A) or fold change, relative to wild type controls (B). Data were analyzed by twoway ANOVA. ${ }^{c} P<0.01$ vs controls. ${ }^{f} P<0.01$ vs NMDA. ${ }^{i} P<0.01$ vs wild type NMDA.

Wild type and hENT1 transgenic neurons were treated with an excitotoxic condition, $100 \mu \mathrm{mol} / \mathrm{L}$ NMDA for $30 \mathrm{~min}$, and extracellular adenosine levels were determined (Figure 4B). NMDA treatment of hENT1 transgenic neurons led to a $75 \%$ increase in extracellular adenosine levels, relative to the same treatment of wild type neurons. As previously observed with rat neurons ${ }^{[22]}$, this increase in adenosine was abolished by treatment with dipyridamole, indicating that nucleoside transporters mediated adenosine release from neurons. Again, it was noted that there was a mismatch between the 15 -fold increase in ENT1 density in synaptosomal membranes and the $75 \%$ increase in adenosine release from cultured neurons evoked by NMDA. This is largely explained by the finite quantities of intracellular purines; treatment with NMDA causes release of upwards of $20 \%$ of radiolabelled purines from these cells.

\section{Hippocampal slice electrophysiology as a bioassay for adenosine production}

Hippocampal slices from rats and mice have been used to record excitatory post-synaptic potentials for many years. Using this method, it has been demonstrated that basal adenosine levels inhibit synaptic activity via activation of adenosine $\mathrm{A}_{1}$ receptors ${ }^{[26]}$. Exogenous adenosine and receptor agonists decrease synaptic activity whereas receptor antagonists increase synaptic activity ${ }^{[26]}$.

Hypoxia and oxygen-glucose deprivation decrease synaptic activity in hippocampal slices ${ }^{[27,28]}$. Adenosine $\mathrm{A}_{1}$ receptor antagonists attenuate this synaptic inhibition ${ }^{[28]}$. This synaptic inhibition is also attenuated in slices from $\mathrm{A}_{1}$ receptor knock out mice ${ }^{[29]}$. Thus, conditions such as hypoxia increase extracellular adenosine levels and promote adenosine $\mathrm{A}_{1}$ receptor activity. Using an enzyme-based biosensor, Frenguelli and colleagues have demonstrated that extracellular adenosine levels rapidly increase in hippocampal slices exposed to ischemic conditions; increases in extracellular ATP were also observed, but were slower and more modest than the increases in adenosine ${ }^{[30]}$. The effect of calcium on adenosine and ATP release evoked by ischemic conditions was examined. Adenosine release was increased, but ATP release was reduced, in calcium-free aCSF containing EGTA, relative to physiological aCSF. Furthermore, ischemia-evoked ATP release was enhanced, but adenosine release was not significantly altered, by treatment with kynurenic acid, a non-selective antagonist of ionotropic glutamate receptors. In sum, these data suggest that the ischemia-evoked release of adenosine is largely independent of ATP release ${ }^{[30]}$.

Nucleoside transport inhibitors produce synaptic inhibition that is blocked by adenosine $A_{1}$ receptor antagonists ${ }^{[26]}$ and these inhibitors were shown to increase adenosine release without an effect on ATP release from ischemic slices ${ }^{[30]}$, indicating that these transporters are more important for adenosine uptake than adenosine release during physiological and hypoxic/ischemic conditions.

Neuronal ENTs in hippocampus mediate adenosine uptake not release during hypoxia/ischemia

In light of research showing that nucleoside transport inhibitors enhance the actions of adenosine in hippocampal slices ${ }^{[24,26]}$ yet in neuron cultures these inhibitors prevent evoked release 
of adenosine ${ }^{[18,20]}$ (Figure 4), we tested adenosine-dependent responses to basal, hypoxic or ischemia-like conditions in hippocampal slices from hENT1 transgenic, relative to wild type, mice.

Using hippocampal slices from wild type mice, it was noted that exogenous adenosine inhibited synaptic transmission with an $\mathrm{IC}_{50}$ value of approximately $25 \mu \mathrm{mol} / \mathrm{L}$; however, using slices from hENT1 transgenic mice, adenosine had an $\mathrm{IC}_{50}$ value of greater than $50 \mu \mathrm{mol} / \mathrm{L}^{[24]}$. In contrast, the adenosine $\mathrm{A}_{1}$ receptor agonist, $N^{6}$-cyclopentyladenosine had similar inhibitory potency in slices from wild type and hENT1 transgenic slices. Furthermore, the $\mathrm{A}_{1}$ receptor antagonist 8-cyclopentyl-1,3-dipropyl-xanthine increased synaptic activity in wild type slices but had no stimulatory effect in hENT1 transgenic slices, indicating that basal adenosine levels were reduced in transgenic slices. In slices exposed to hypoxic or oxygen-glucose deprivation conditions, hENT1 transgenic slices showed less synaptic inhibition than wild type slices (Figure 5). NBTI $(100 \mathrm{nmol} / \mathrm{L})$ decreased synaptic activity in slices from both wild type and hENT1 transgenic mice and enhanced the synaptic depression produced by hypoxia or oxygen-glucose deprivation. This effect was greater in slices from hENT1 transgenic mice (Figure 5) as, in the presence of NBTI, wild type and hENT1 transgenic slices responded similarly to hypoxia or oxygen-glucose deprivation. Together, these data indicate that hENT1 expression in hippocampal neurons was associated with reduced extracellular adenosine levels, increased clearance of extracellularly applied adenosine, and reduced adenosine receptor activation in response to hypoxia or ischemia-like conditions.

\section{Neuronal hENT1 expression is associated with larger endothelin-1 induced cerebral infarcts}

Endothelin-1 is a potent vasoconstrictor that has been used to produce ischemic infarcts ${ }^{[31]}$. Previously, it has been determined that reduction of blood flow to $20 \%$ of normal flow results in ischemic injury ${ }^{[32]}$. We found that an injection of endothelin-1 (400 pmol) into cerebral cortex produced a longlasting decrease in blood flow at the site of injection ${ }^{[33]}$. Using perfusion-weighted magnetic resonance imaging, cerebral blood flow was determined at 4 and $48 \mathrm{~h}$ post-injection and found to be less than $15 \%$ and $50 \%$, respectively, relative to pre-injection values. Volume matched saline injections produced transient decreases in cerebral blood flow that returned to pre-injection values within $48 \mathrm{~h}$.

Interestingly, while cerebral blood flow responses were similar between wild type and hENT1 transgenic mice, cerebral infarct sizes were $60 \%$ larger, as determined by T2-weighted magnetic resonance imaging, in hENT1 transgenic mice ${ }^{[33]}$. This genotype difference in infarct sizes was not evident in mice that had received the adenosine receptor antagonist caffeine $(25 \mathrm{mg} / \mathrm{kg}$, ip) $30 \mathrm{~min}$ prior to intracerebral injection of endothelin-1, indicating that a genotype difference in ischemic adenosine levels and adenosine receptor activity was responsible for the differences in ischemic infarct sizes between wild type and hENT1 transgenic mice ${ }^{[33]}$.
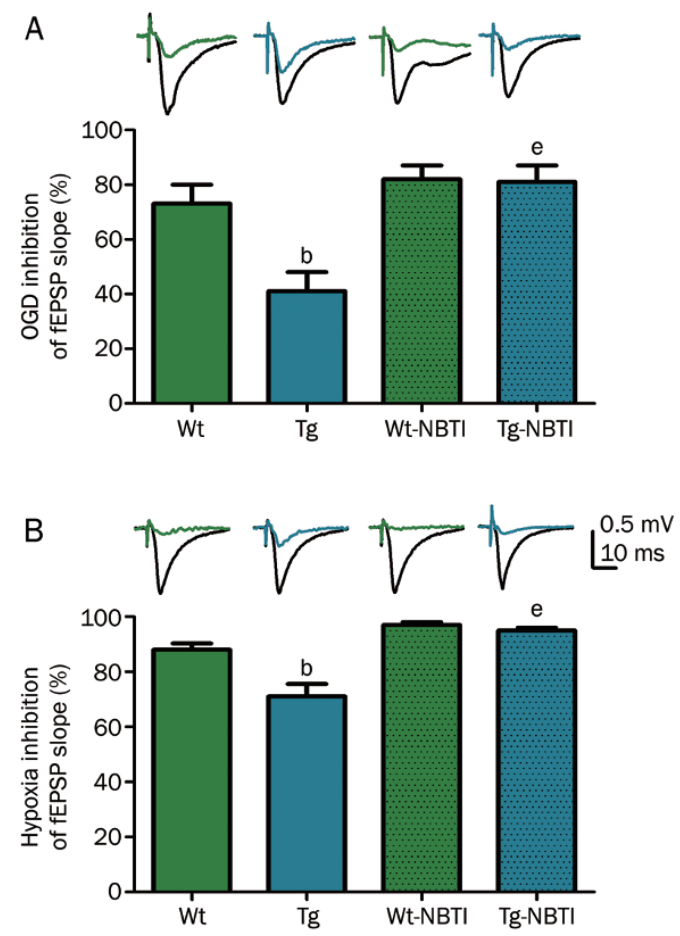

Figure 5. Extracellular post-synaptic excitatory potentials (fEPSP) were recorded from electrically stimulated hippocampal slices from wild type (Wt) or hENT1 transgenic (Tg) mice. Synaptic responses were evoked by stimulation of the Schaffer collateral/commissural pathway with a concentric bipolar stimulating electrode with $0.1 \mathrm{~ms}$ pulse width at $30 \mathrm{~s}$ intervals. Extracellular field excitatory postsynaptic potentials (fEPSPs) were recorded in striatum radiatum of CA1 hippocampus using glass microelectrodes (1-2 $\Omega$ ) filled with aCSF. Slices were continuously superfused with aCSF or with $100 \mathrm{nmol} / \mathrm{L} \mathrm{S}$-(p-nitrobenzyl)-6-thioinosine (NBTI) in aCSF at a flow rate of $1.5 \mathrm{~mL} / \mathrm{min}\left(32.5^{\circ} \mathrm{C}\right)$. (A) Slices were exposed to glucosefree hypoxic aCSF (oxygen-glucose deprivation; OGD) for 3 min. (B) Slices were exposed to hypoxic aCSF for $10 \mathrm{~min}$. Representative wave forms show fEPSP response before (black) or during OGD (A) or hypoxia (B) from Wt (green) or Tg (blue) slices. Bars are mean \pm SEM $(n \geq 3)$ of maximally inhibited fEPSP responses, calculated as the difference between black and colored waveforms. Data were analyzed by one-way ANOVA and Bonferroni post-tests. ${ }^{b} P<0.05$ vs Wt. ${ }^{e} P<0.05$ vs Tg. Data are adapted from ${ }^{[24]}$; for additional details on methods, see ${ }^{[24]}$.

\section{Conclusions from ENT1 null and hENT1 transgenic mice}

Adenosine dependent activation of $\mathrm{A}_{1}$ receptors is beneficial in cerebral ischemia ${ }^{[34]}$. It has been repeatedly demonstrated that activation of $A_{1}$ receptors causes a rapid and profound decrease in synaptic activity with hypoxia or oxygen-glucose deprivation $^{[26,28,35,36]}$. Nevertheless, the origin of this adenosine is still under investigation.

ENT1 is an equilibrative transporter that transports adenosine across cell membranes in a direction dictated by its concentration gradient. We proposed that, in neurons and brain tissues from mice with enhanced neuronal expression of ENT1, elevated extracellular adenosine levels and enhanced adenosine $A_{1}$ receptor activity would be observed if adenosine was primarily produced intracellularly, in accordance with the tra- 

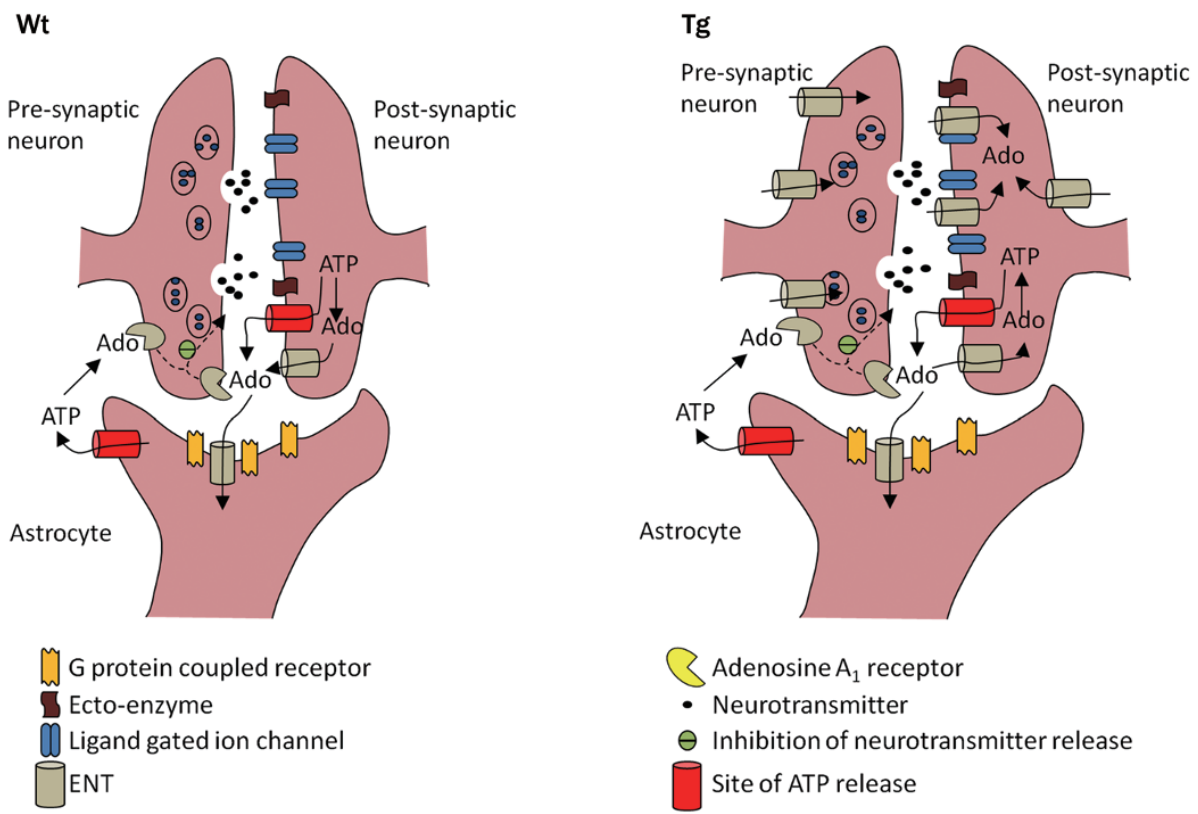

Figure 6. A schematic diagram illustrating the effect of neuronal expression of human equilibrative nucleoside transporter 1 (hENT1). While mouse ENT1 and ENT2 are located on neurons and astrocytes, hENT1 is selectively expressed in neurons. From the data obtained with hippocampal slice electrophysiology and endothelin-1 injections into cerebral cortex, it appears that hENT1 expression in neurons leads to increased neuronal uptake of adenosine during hypoxic-ischemic conditions and decreased extracellular adenosine levels leading to decreased adenosine $\mathrm{A}_{1}$ receptor activity. Ado, adenosine. Wt: wild type littermates. Tg: hENT1 transgenic mice.

ditional view of adenosine acting as a retaliatory metabolite. Interestingly, our studies reveal a difference between cultured neurons and hippocampal slices. The neuronal cultures exhibited nucleoside transporter mediated adenosine release during conditions of excitotoxicity but the hippocampal slices exhibited neuronal transporter mediated adenosine uptake during hypoxia/ischemia. The in vivo stroke model was consistent with the hippocampal slice experiments, as stroke injury was enhanced in hENT1 transgenic mice, which would be expected if adenosine levels and adenosine $A_{1}$ receptor activity were reduced (Figure 6).

From our results, we conclude that when fetal neurons are cultured in isolation of other cell types, they appear to efflux cytoplasmic adenosine, produced from intracellular ATP that is rapidly degraded during conditions of hypoxia or ischemia. However, this is not the primary source of adenosine in in vitro hippocampus or in vivo cerebral cortex, derived from adult animals. Interestingly, adenosine levels and adenosine receptor activity were decreased in nucleus accumbens from ENT1 knock out mice, indicating that ENT1 mediates net adenosine efflux in this brain region ${ }^{[16,17]}$. Further research is required to resolve these differences between pathways that produce adenosine in cultured neurons and in different regions of adult brain. As cytosolic formation of adenosine in neurons is not a primary source of adenosine under basal, hypoxic or ischemic experimental conditions in adult cerebral cortex or hippocampus, current studies are underway to evaluate alternative sources of extracellular adenosine, including ATP, or other adenine nucleotides, released from neurons or glia and metabolized to adenosine extracellularly.

\section{Acknowledgements}

Research in the authors' laboratories is supported by the Canadian Institutes for Health Research, the Heart and Stroke Foundation of Manitoba, the Natural Sciences and Engineering Research Council of Canada and the St Boniface General Hospital Research Foundation. BCA is a Research Affiliate at the University of Manitoba's Centre on Aging and the Everett Endowment Fund Chair.

\section{References}

1 Camici M, Micheli V, Ipata PL, Tozzi MG. Pediatric neurological syndromes and inborn errors of purine metabolism. Neurochem Int 2010; 56: 367-78.

2 Dunwiddie TV, Masino SA. The role and regulation of adenosine in the central nervous system. Annu Rev Neurosci 2001; 24: 31-55.

3 Newby AC, Worku Y, Holmquist CA. Adenosine formation. Evidence for a direct biochemical link with energy metabolism. Adv Myocardiol 1985; 6: 273-84.

4 Phillis JW. The effects of selective A1 and A2a adenosine receptor antagonists on cerebral ischemic injury in the gerbil. Brain Res 1995; 705: 79-84.

5 Melani A, Pantoni L, Bordoni F, Gianfriddo M, Bianchi L, Vannucchi MG, et al. The selective A2A receptor antagonist SCH 58261 reduces striatal transmitter outflow, turning behavior and ischemic brain damage induced by permanent focal ischemia in the rat. Brain Res 2003; 959: 243-50.

6 McCullough LD, Zeng Z, Li H, Landree LE, McFadden J, Ronnett GV. Pharmacological inhibition of AMP-activated protein kinase provides neuroprotection in stroke. J Biol Chem 2005; 280: 20493-502. 
7 Hardie DG, Ross FA, Hawley SA. AMPK: a nutrient and energy sensor that maintains energy homeostasis. Nat Rev Mol Cell Biol 2012; 13: 251-62.

8 Li J, Zeng Z, Viollet B, Ronnett GV, McCullough LD. Neuroprotective effects of adenosine monophosphate-activated protein kinase inhibition and gene deletion in stroke. Stroke 2007; 38: 2992-9.

9 Pascual O, Casper KB, Kubera C, Zhang J, Revilla-Sanchez R, Sul JY, et al. Astrocytic purinergic signaling coordinates synaptic networks. Science 2005; 310: 113-6.

10 Zhang JM, Wang HK, Ye CQ, Ge W, Chen Y, Jiang ZL, et al. ATP released by astrocytes mediates glutamatergic activity-dependent heterosynaptic suppression. Neuron 2003; 40: 971-82.

11 Huxtable AG, Zwicker JD, Poon BY, Pagliardini S, Vrouwe SQ, Greer JJ, et al. Tripartite purinergic modulation of central respiratory networks during perinatal development: the influence of ATP, ectonucleotidases, and ATP metabolites. J Neurosci 2009; 29: 14713-25.

12 Burnstock G. Purinergic signalling and disorders of the central nervous system. Nat Rev Drug Discov 2008; 7: 575-90.

13 Parkinson FE, Damaraju VL, Graham K, Yao SYM, Baldwin SA, Cass $\mathrm{CE}$, et al. Molecular biology of nucleoside transporters and their distributions and functions in the brain. Curr Top Med Chem 2011; 11: 948-72.

14 Baldwin SA, Yao SY, Hyde RJ, Ng AM, Foppolo S, Barnes K, et al. Functional characterization of novel human and mouse equilibrative nucleoside transporters (hENT3 and mENT3) located in intracellular membranes. J Biol Chem 2005; 280: 15880-7.

15 Barnes K, Dobrzynski H, Foppolo S, Beal PR, Ismat F, Scullion ER, et al. Distribution and functional characterization of equilibrative nucleoside transporter-4, a novel cardiac adenosine transporter activated at acidic pH. Circ Res 2006; 99: 510-9.

16 Choi DS, Cascini MG, Mailliard W, Young H, Paredes P, McMahon T, et al. The type 1 equilibrative nucleoside transporter regulates ethanol intoxication and preference. Nat Neurosci 2004; 7: 855-61.

17 Nam HW, Lee MR, Zhu Y, Wu J, Hinton DJ, Choi S, et al. Type 1 equilibrative nucleoside transporter regulates ethanol drinking through accumbal $N$-methyl-D-aspartate receptor signaling. Biol Psychiatry 2011; 69: 1043-51.

18 Rose JB, Naydenova Z, Bang A, Ramadan A, Klawitter J, Schram K, et al. Absence of equilibrative nucleoside transporter 1 in ENT1 knockout mice leads to altered nucleoside levels following hypoxic challenge. Life Sci 2011; 89: 621-30.

19 Rose JB, Naydenova Z, Bang A, Eguchi M, Sweeney G, Choi DS, et al. Equilibrative nucleoside transporter 1 plays an essential role in cardioprotection. Am J Physiol Heart Circ Physiol 2010; 298: H771-7.

20 Parkinson FE, Xiong W. Stimulus- and cell-type-specific release of purines in cultured rat forebrain astrocytes and neurons. J Neurochem 2004; 88: 1305-12.

21 Zamzow CR, Xiong W, Parkinson FE. Adenosine produced by neurons is metabolized to hypoxanthine by astrocytes. J Neurosci Res 2008; 86: 3447-55.

22 Zamzow CR, Xiong W, Parkinson FE. Astrocytes affect the profile of purines released from cultured cortical neurons. J Neurosci Res 2008; 86: 2641-9.

23 Parkinson FE, Xiong W, Zamzow CR, Chestley T, Mizuno T, Duckworth ML. Transgenic expression of human equilibrative nucleoside transporter 1 in mouse neurons. J Neurochem 2009; 109: 562-72.

24 Zhang D, Xiong W, Albensi BC, Parkinson FE. Expression of human equilibrative nucleoside transporter 1 in mouse neurons regulates adenosine levels in physiological and hypoxic-ischemic conditions. J Neurochem 2011; 118: 4-11.

25 Etherington LA, Patterson GE, Meechan L, Boison D, Irving AJ, Dale N, et al. Astrocytic adenosine kinase regulates basal synaptic adenosine levels and seizure activity but not activity-dependent adenosine release in the hippocampus. Neuropharmacology 2009; 56: 429-37.

26 Dunwiddie TV, Diao L. Extracellular adenosine concentrations in hippocampal brain slices and the tonic inhibitory modulation of evoked excitatory responses. J Pharmacol Exp Ther 1994; 268: 537 45.

27 Frenguelli BG, Llaudet E, Dale N. High-resolution real-time recording with microelectrode biosensors reveals novel aspects of adenosine release during hypoxia in rat hippocampal slices. J Neurochem 2003; 86: 1506-15.

28 Pearson T, Damian K, Lynas RE, Frenguelli BG. Sustained elevation of extracellular adenosine and activation of $A$ receptors underlie the post-ischaemic inhibition of neuronal function in rat hippocampus in vitro. J Neurochem 2006; 97: 1357-68.

29 Johansson B, Halldner L, Dunwiddie TV, Masino SA, Poelchen W, Gimenez-Llort L, et al. Hyperalgesia, anxiety, and decreased hypoxic neuroprotection in mice lacking the adenosine $\mathrm{A}_{1}$ receptor. Proc Natl Acad Sci U S A 2001; 98: 9407-12.

30 Frenguelli BG, Wigmore G, Llaudet E, Dale N. Temporal and mechanistic dissociation of ATP and adenosine release during ischaemia in the mammalian hippocampus. J Neurochem 2007; 101 : 1400-13.

31 Windle V, Szymanska A, Granter-Button S, White C, Buist R, Peeling J, et al. An analysis of four different methods of producing focal cerebral ischemia with endothelin-1 in the rat. Exp Neurol 2006; 201 : 324-34.

32 Siesjo BK. Pathophysiology and treatment of focal cerebral ischemia. Part I: Pathophysiology. J Neurosurg 1992; 77: 169-84.

33 Soylu H, Zhang D, Buist R, Martin M, Albensi BC, Parkinson FE. Intracortical injection of endothelin-1 induces cortical infarcts in mice: effect of neuronal expression of an adenosine transporter. Exp Transl Stroke Med 2012; 4: 4.

34 Rudolphi KA, Schubert P, Parkinson FE, Fredholm BB. Adenosine and brain ischemia. Cerebrovasc Brain Metab Rev 1992; 4: 346-69.

35 Masino SA, Diao L, Illes P, Zahniser NR, Larson GA, Johansson B, et al. Modulation of hippocampal glutamatergic transmission by ATP is dependent on adenosine a(1) receptors. J Pharmacol Exp Ther 2002; 303: 356-63.

36 Parkinson FE, Xiong W, Zamzow CR. Astrocytes and neurons: different roles in regulating adenosine levels. Neurol Res 2005; 27: 153-60. 